\title{
Left Atrial Size and Left Ventricular Mass Index in Hypertensive Patients
}

\author{
Kartikesh Kumar Thakur ${ }^{1}$, Ratna Mani Gajurel ${ }^{1}$, Sheelendra Shakya ${ }^{1}$, Aseem Bhattarai ${ }^{2}$, Mithileswor Raut ${ }^{2}$, Arun \\ Sayami $^{1}$
}

Affiliations:

${ }^{1}$ Department of Cardiology, Manmohan Cardiothoracic Vascular and Transplant Centre, Kathmandu

${ }^{2}$ Department of Biochemistry, Institute of Medicine, Maharjgunj, Kathmandu

Correspondence to:

Kartikesh Kumar Thakur

Manmohan Cardiothoracic Vascular and Transplant

Kathmandu, Nepal

Email: kartikesh_143@hotmail.com

How to cite this article:

Thakur KK, Gajurel RM, Shakya S, Bhattarai A, Raut M,Sayami A. Left atrial size and left ventricular mass index in hypertensive patients. Ann. Clin. Chem. Lab. Med. 2016:2(2);21-25

DOI: http://dx.doi.org/10.3126/acclm.v2i2.15035

(c) 2016 Nepalese Association for Clinical Chemistry

\section{(c) (1) ()}

This work is licensed under a Creative Commons Attribution-ShareAlike 4.0 International License.

\section{Abstract}

\section{BACKGROUND}

This study was conducted with the objective of evaluation of the occurrence of left atrial enlargement (LAE) in hypertensive patients and its relationship with left ventricular mass index (LVMI).

\section{METHODS}

This single centre study was carried out at the Manmohan Cardiothoracic Vascular and Transplant Center, where total of 99 patients with hypertension who had left ventricular hypertrophy on echocardiography defined by left ventricular septal wall thickness $>9 \mathrm{~mm}$ for female and $>10$ $\mathrm{mm}$ for male, were selected from May 2015 to August 2015.

\section{RESULTS}

In the study, the left atrium was enlarged in $31 \%$ of the selected patients. A total of $44 \%$ of patients having left atrial enlargement had increased left ventricular mass index as well, while a positive correlation $(r=0.3)$ was observed between the left atrial size and the left ventricular mass index $(p=0.001)$. The mean Left Ventricular Mass Index was $98 \pm 32.7 \mathrm{~g} / \mathrm{m}^{2}$ as compared to $115.34 \pm 40.2$ in patients with left atrial enlargement. The mean systolic blood pressure with left atrial enlargement was $146.70 \pm 21.64 \mathrm{~g} / \mathrm{m}^{2}$. Out of 99 patients, $80 \%$ of patients with left atrial enlargement had elevated body mass indexes, $48 \%$ had a history of smoking, 32\% had diabetes mellitus, $22 \%$ had other complications of hypertension, and $12 \%$ had dyslipidemia.

\section{CONCLUSIONS}

We concluded that the Left Atrial Size correlates significantly with the Left Ventricular Mass Index. In addition, the presence of left atrial enlargement should raise the suspicion of other metabolic derangements in hypertensive patients.

KEY WORDS: Hypertension, Left atrial enlargement (LAE), Left ventricular mass index 
Multiple studies have established till date that changes in left ventricular structure and function, evaluated by indicators such as left ventricular mass (LVM), left ventricular mass index (LVMI) and an impairment of diastolic or systolic function is a common implication of long standing hypertension[1,2].Another important indicator, however, is the left atrial size, an anatomic alteration of which has been associated independently with the occurrence of heart failure [3], hypertensive heart disease and other cardiovascular event $[4,5]$. The enlargement of the left atrium is commonly seen in association with left ventricular hypertrophy and an overall increase in left ventricular mass [6] but the independent risks related to cardiac function (apart from known associations with a chronically elevated LV filling pressure and diastolic dysfunction even in the absence of mitral valve disease) that are contributed in part by the increased left atrial size, yet remains unexplored.

The aim of the present study is to evaluate the left atrial size, portrayed fairly by $\mathrm{m}$-mode LA dimensions (precisely, the AP diameter $[6,8]$ in relation to the left ventricular mass and left ventricular mass index of patients with long standing hypertension. This will help establish left atrial size as an independent cardiac event associated with hypertension, in addition to known complications like left ventricular hypertrophy and diastolic dysfunction.

\section{Methods}

This cross sectional study was conducted at the Manmohan Cardiothoracic Vascular and Transplant Center between May-August 2015. Ninty-nine hypertensive patients (meeting the JNC VII criteria for hypertension) and more than 18 years of age visiting the Out Patients Department were selected for the study. Information on presenting complaints (chest pain, dyspnea) and risk factors (DM, Hypertension or dyslipidemia) were obtained from a medical history. All of these patients underwent a complete physical and echocardiographic examination at the time of evaluation and the parameters obtained were, 1) Blood Pressure (systolic and diastolic, brachial, sitting position after 5 minutes of rest), 2) Height ( $m$ ) and Weight $(\mathrm{kg})$ for calculation of Body Mass Index. Patients with known pericardial effusion, left bundle branch block, left anterior hemiblock, pre-excitation, acute myocardial events were excluded from the study.

\section{Echocardiographic Variables}

Images were obtained in left parasternal long axis and short axis views with patients in the left lateral position using VIVID7 ${ }^{\circ}$ echocardiography machine. Only tests with proper interface visualization that showed simultaneous images of septum, LV internal diameter and posterior wall were considered adequate for determination of the LV mass. LA diameter was assessed using a leading edge to leading edge measurement of the maximal diameter starting from the posterior aortic root to the posterior LA wall at end systole [9]. LV measurements were taken according to the recommendation of the American Society of Echocardiography (ASE), with the M-mode guided by 2-D mode. LV systolic and diastolic diameters, interventricular septal and LV posterior wall thickness were measured at end diastole. All the values were recorded in millimeters $(\mathrm{mm})$. LV mass was calculated using corrected ASE formula: $L V M=$ $0.8\left\{1.04\left[(I V S+L V D d+P W T)^{3}-L V D d^{3}\right]+0.6,{ }^{9}\right.$ where IVS stands for interventricular septum, LVDd for LV diastolic diameter, and PWT for posterior wall thickness. LV mass index was calculated by dividing LV mass by BSA. LAE and LV hypertrophy (LVH) were defined as Left atrial AP dimension $>38 \mathrm{~mm}$ (females) and $>40 \mathrm{~mm}$ (males) and LV mass index (LVMI) $>95 \mathrm{~g} / \mathrm{m}^{2}$ (females) and $115 \mathrm{~g} / \mathrm{m}^{2}$ (Males) respectively. [9]

\section{Statistical Analysis}

The data was entered and analyzed using IBM SPSS ${ }^{\circ}$ 20. The variables studied were age, gender, diabetes, hypertension, clinical findings (height, weight, BMI), LV mass, LV mass index, and Left Atrial Size (based on LA diameter). Mean \pm S.D. were calculated for age, BMI, LV Mass and LV mass index. Gender and risk factors were expressed as frequency distribution tables. Bivariate Correlations were used to observe the association between left atrial size and left ventricular mass index. 


\section{Results}

Out of the 99 patients studied, mean age of the study population ( $n=99)$ was $56.65 \pm 13.24$ and majority of the patients were in age group 50 - 70. Male to female ratio was 2:1. Mean body mass index of the patients was $25.51 \pm 3.5$; the mean systolic blood pressure was $150.23 \pm 21.84$. Only $13 \%$ of patients had normal body weight according to BMI.

An enlarged LA was present in 31\% (35\% of Females and $29 \%$ of Males) of the study group. An increased left ventricular mass index was present in $44 \%$ of the patients that also had a LA enlargement. There was a moderate positive correlation between LA enlargement and left ventricular mass index $(r=0.3$, $p<0.001)$. The Mean LV mass index was $98 \pm 32.7$ in the selected patients as compared to $115.34 \pm 40.2$ in patients with left atrial enlargement. Mean systolic blood pressure with LA enlargement was $146.70 \pm$ 21.64. 80\% of patients with LA enlargement had more than normal body weight by BMI, $8 \%$ had a history of smoking, $32 \%$ had diabetes mellitus, 22\% had complications of HTN and $12 \%$ had dyslipidemia.

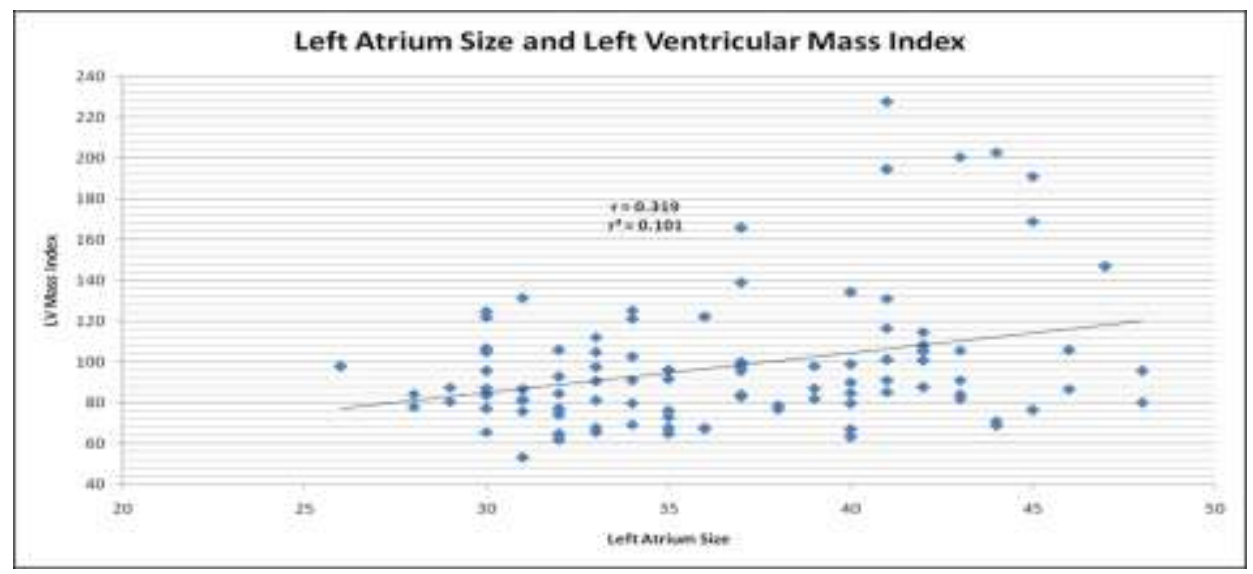

Figure1.Left atrial size and left ventricular mass index

Table2.Genderwise distribution of LV mass, LV massindex and left atrial diameter

\begin{tabular}{lccc|}
\hline Category & LV Mass Index & LV Mass & Left Atrial AP Diameter \\
\hline Male & $98.15 \pm 31.65$ & $167.6 \pm 45.57$ & $36.87 \pm 5.5$ \\
Female & $97.72 \pm 35.2$ & $152.27 \pm 52.3$ & $36.14 \pm 5.07$ \\
Total & $98.00 \pm 32.7$ & $162.39 \pm 48.28$ & $36.62 \pm 5.36$ \\
\hline
\end{tabular}

\section{Discussion}

Multiple studies have shown that the left atrial enlargement is an independent long term predictor of cardiovascular events [10]. Although various factors like age, race, body mass index, duration of hypertension, valvular heart disease influence the left atrial size [11]. The enlargement and altered function of the left atrium (LA)are associated with an increased prevalence of atrial fibrillation and stroke in men and death in both genders $[12,13]$.

It has recently been demonstrated that LA dimension $>=4.17 \mathrm{~cm}$ is significantly associated with increased risk of death and hospitalization." Moreover, electrocardiographic (ECG) and echocardiographic investigations have shown LA enlargement in patients with hypertension [14]. 
In STS(strong heart study) concluded that LA diameter independently predicted incident cardiovascular event in middle aged and elderly adults [4].

Our study showed that enlarged LA was present in $31 \%$ ofthe study group which is similar to the study done by M. A. Tedesco et al in a study "Left atrial Size in 164 Hypertensive Patients: An Echocardiographic and Ambulatory Blood Pressure Study" which showed that the left atrial enlargement was present in $35 \%$ of elderly and in $24 \%$ of younger patients $(p=0.31)$ [14].

Cuspidi et. al [15] in their study "Prevalence of Echocardiographic Left-Atrial Enlargement in Hypertension: A Systematic Review of Recent Clinical Studies" also found that $32 \%$ of patients had LA enlargement.

In our study the increased left ventricular mass index was present in $44 \%$ of the patients that also had a left atrial enlargement. There was a moderate positive correlation between left atrial enlargement and left ventricular mass index $(r=0.3, p<0.001)$.

M. A. Tedesco et. al in their study concluded that LA enlargement has significant association with age and left ventricular mass index [14]. Similar findings with significant positive correlation between LA enlargement and left ventricular mass has been shown in LIFE study [16].

In our study $35 \%$ of female and $29 \%$ of male had LA enlargement similar to the study done Gertds E. Oikari et al [16] in Losartan Intervention For Endpoint

\section{REFERENCES}

1. Franklin SS WK, Papademetriou Cardiovascular morbidity and mortality in hypertensive patients with lower versus higher risk: a LIFE substudy. Hypertension 2005;2005;46(3): (3):492-9.

2. Bombelli M, Facchetti R, Carugo S, Madotto F, Arenare F, Quarti-Trevano F, et al. Left ventricular hypertrophy increases cardiovascular risk independently of in-office and out-of-office blood pressure values. Journal of hypertension. 2009;27(12):2458-64.

3. Gardin JM, McClelland R, Kitzman D, Lima JA, Bommer W, Klopfenstein HS, et al. M-mode echocardiographic predictors of six-to seven-year incidence of coronary heart disease, stroke, congestive heart failure, and mortality in an elderly cohort (the Cardiovascular Health Study). The American journal of cardiology. 2001;87(9):1051-7.

4. Kizer JR, Bella JN, Palmieri V, Liu JE, Best LG, Lee ET, et al. Left atrial diameter as an independent predictor of first clinical
Reduction in Hypertension (LIFE) Study. Correlates of left atrial size in hypertensive patients with left ventricularhypertrophy study suggesting that LA enlargement is common in female possibly due to altered left ventricular geometry.

Our study showed that increased other parameters of metabolic syndrome and coronary risk factor besides hypertension.

In our study, $80 \%$ of patients with LA enlargement had more than normal body weight by BMI, $8 \%$ had a history of smoking, $32 \%$ had diabetes mellitus, $22 \%$ had complications of HTN and $12 \%$ had dyslipidemia. These findings are supported by the PAMELA study which showed that a LA diameter exhibited a significant direct relationship with several variables (age, LVM, body mass index and serum total cholesterol, triglycerides, and glucose) while showing a significant inverse relationship with few others (serum high-density lipoprotein cholesterol ). [10]

\section{Conclusions}

The Left Atrial Size correlates significantly with the Left Ventricular Mass Index in the context of hypertensive patients. In addition, the presence of left atrial enlargement should raise the suspicion of other metabolic derangements in hypertensive patients. Prospective studies with larger sample sizes need to be conducted in the future to establish the cardiac implications associated with left atrial enlargement.

\section{Conflicts of interest}

None declared. cardiovascular events in middle-aged and elderly adults: the Strong Heart Study (SHS). American heart journal. 2006;151(2):412-8.

5. Tsang TS, Barnes ME, Gersh BJ, Takemoto Y, Rosales AG, Bailey $\mathrm{KR}$, et al. Prediction of risk for first age-related cardiovascular events in an elderly population: the incremental value of echocardiography. Journal of the American College of Cardiology. 2003;42(7):1199-205.

6. Cuspidi C, Negri F, Sala C, Valerio C, Mancia G. Association of left atrial enlargement with left ventricular hypertrophy and diastolic dysfunction: a tissue Doppler study in echocardiographic practice. Blood pressure. 2012;21(1):24-30.

7. Mureddu GF, Cioffi G, Stefenelli C, Boccanelli A. Relationships of the appropriateness of left ventricular mass to left atrial size and function in arterial hypertension. Journal of Cardiovascular Medicine. 2007;8(6):445-52. 
8. Cioffi G, Mureddu GF, Stefenelli C, de Simone G. Relationship between left ventricular geometry and left atrial size and function in patients with systemic hypertension. Journal of hypertension. 2004;22(8):1589-96.

9. Lang RM, Badano LP, MorAvi V, Afilalo J, Armstrong A, Ernande $L$, et al. Recommendations for cardiac chamber quantification by echocardiography in adults: an update from the American Society of Echocardiography and the European Association of Cardiovascular Imaging. Journal of the American Society of Echocardiography. 2015;28(1):1-39. e14.

10. Michele Bombelli RF, Cesare Cuspidi, Paolo Villa, Dario Dozio, Gianmaria Brambilla, Guido Grassi, Giuseppe Mancia. Prognostic Significance of Left Atrial Enlargement in a General Population Results of the PAMELA Study. Hypertension. 2014;64:1205-11.

11. BJ GJRDWDM. : Left atrial size in hypertensive men: Intluence ofobesity, race and ag. J Am Coll Cirrdiol 1997;29:651-8.

12. Benjamin EJ, D'Agostino RB, Belanger AJ, Wolf PA, Levy D. Left atrial size and the risk of stroke and death The Framingham Heart Study. Circulation. 1995;92(4):835-41.

13. Cabin HS, Clubb KS, Hall C, Perlmutter RA, Feinstein AR. Risk for systemic embolization of atrial fibrillation without mitral stenosis. The American journal of cardiology. 1990;65(16):1112-6.

14. Michele A. Tedesco GDS, Gennaro FN, Diana larussi, Do lacono, MD.. Fesc Left Atrial Size in 164 Hypertensive Patients: An Echocardiographic and Ambulatory Blood Pressure Study ClinCardiol. 2001;24:603-7.

15. Cesare Cuspidi, 2 Marta Rescaldani,3 and Carla Sala3. Prevalence of Echocardiographic Left-Atrial Enlargement in Hypertension: A Systematic Review of Recent Clinical Studies. American Journal of Hypertension. 2013;26(4)

16. Gerdts E, Oikarinen L, Palmieri V, Otterstad J, Wachtell K, Boman K, et al. Losartan Intervention For Endpoint Reduction in Hypertension (LIFE) Study. Correlates of left atrial size in hypertensive patients with left ventricular hypertrophy: The losartan intervention for endpoint reduction in hypertension (LIFE) study. Hypertension. 2002;39(3):739-43. 